\title{
Next-Generation PET Capability with Lutetium Fine Silicate and Multi-Pixel Photon Counter
}

\author{
Makoto Yamazaki, ${ }^{a}{ }^{*}$ Tohru Takeshita ${ }^{\mathrm{b}}$, Yoji Hasegawa ${ }^{\mathrm{b}}$ \\ a.Interdisciplinary Graduate School of Science and Engineering, Shinshu University, \\ 3-1-1 Asahi, Matsumoto, Japan \\ b.Faculty of Science, Department of Physics, Shinshu University, \\ E-mail: yamazaki@azusa. shinshu-u.ac.jp
}

\begin{abstract}
Currently, positron emission tomography (PET) systems are rapidly developing owing to the invention of new scintillators and photosensors, which contribute to construction of clearer and high-resolution images in cancer diagnosis. In this study, we used a Lutetium Fine Silicate (LFS) inorganic scintillator and multi-pixel photon counter (MPPC) as a photosensor. This combination is realistic for next-generation PET systems. These devices were directly coupled to form a unit detector. A pair of detector units was placed face-to-face, and they simultaneously detected annihilation gamma rays, assuming a pair detector in the PET system. A timing resolution of $96 \mathrm{ps}$ in full width at half maximum (FWHM) was obtained. This result is almost the fastest timing resolution ever achieved. A good timing resolution contributes to noise reduction that leads to clear images. Furthermore, a spatial resolution of $1.9 \mathrm{~mm}$ in FWHM was obtained, which is sufficient to meet the spatial resolution requirements for nextgeneration PET systems.
\end{abstract}

KEYWORDS: Gamma camera, SPECT, PET PET/CT, coronary CT angiography (CTA); Photon detectors for UV, visible and IR photons (solid-state); Scintillators and scintillating fibres and light guides.

${ }^{\mathrm{a} C o r r e s p o n d i n g ~ a u t h o r . ~}$ 


\section{Contents}

1. Introduction 1

2. Materials 2

2.1 Lutetium Fine Silicate 2

2.2 Multi-Pixel Photon Counter (MPPC)

2.2.1 Gain and noise rate 4

3. Experimental Setup 5

4. Results 6

4.1 Energy resolution $\quad 6$

4.2 Timing resolution $\quad 7$

4.2.1 Comparison of timing resolution between LFS and LYSO crystals 7

4.2.2 Dependence of thresholds for timing resolution 9

4.2.3 Mechanisms of jitter and timing resolution 9

$\begin{array}{lr}\text { 4.3 Spatial resolution } & 10\end{array}$

5. Discussion 11

6. Conclusion 12

\section{Introduction}

Time-of-flight (TOF) performance is one of the important features in next-generation positron emission tomography (PET) systems, which can improve images by rejecting accidental coincidence with other photons. Cancer cells are identified by crossing points of many back-toback annihilation gamma rays with energies of $0.511 \mathrm{MeV}$, which mostly emerge from positron annihilation in cancer cells. The positron-emitting nuclei injected in the patient are distributed in the human body and produce photons traveling in all directions. The annihilation gamma rays give many candidate positions for cancer cells by detection within the time window of coincidence, resulting in the degradation of PET image. A high-precision detector can significantly reduce the disturbance due to background photons [1] [2]. To achieve fine TOF capability, we reconsider two basic components, scintillator crystal and photosensor with respect to timing accuracy.

Currently, lutetium yttrium orthosilicate (LYSO) is widely used because of its short scintillation decay time (40 ns) as well as high density. Typically, a PET system with LYSO and photomultiplier tubes has a timing resolution of 500-600 ps. In this study, we introduce the newly available Lutetium Fine Silicate (LFS) [3]. LFS has properties similar to those of LYSO according to the Zecotek Photonics, which manufactures the material. The signal decay time is $36 \mathrm{~ns}$, which is comparable to that of LYSO, and it emits scintillation light of $420 \mathrm{~nm}$ at peak intensity.

In addition, we introduce a newly developed silicon photosensor, which has high gain and fast signal because of the Geiger mode operation in small pixels. The output signal is the sum of signals from all fired pixels. While there are several choices for such pixelated avalanche photodiodes, we adopted the multi-pixel photon counter (S10362-11-025P) from Hamamatsu Photonics K.K. [4]. We have contacted the company to discuss the development of this device on several occasions; therefore, we understand the signal-generation mechanism. This is one of the applications of MPPC to a field other than high-energy physics. There are a couple of 
possibilities, whereby an MPPC can be selected on the basis of the application. The sizes of the detector sensitive area and pixel in the sensitive area are the most critical parameters.

According to simulations, the spatial resolution of the PET system is mostly determined by the scintillator size. For a scintillator with a cross section of $3 \times 3 \mathrm{~mm}^{2}$, the spatial resolution is $\sim 1 \mathrm{~mm}$ which is the maximum limit for positron annihilation phenomena in the human body, due to the deviation of collinearity of the back-to-back photons that are generated by annihilation in flight of a positron. In our experiments, the spatial resolution was measured by placing a ${ }^{22} \mathrm{Na}$ gamma-ray source at the center of a couple of detectors; then, the source is moved orthogonally by $0.50 \mathrm{~mm}$ using a micrometer. The data are counted at each point and fitted by a Gaussian function. According to the fitting, a spatial resolution of $\sim 1 \mathrm{~mm}$ was obtained at FWHM.

A scintillator combination of $3 \times 3 \times 15 \mathrm{~mm}^{3}$ in our experiment and simulations, which are discussed later, can achieve a 1-mm spatial resolution. For the measurements, we adopted a 15$\mathrm{mm}$-long scintillator. The selection of the photosensor is affected by the $3 \times 3 \mathrm{~mm}^{2}$ cross section of the scintillator. Some tested the $3 \times 3 \mathrm{~mm}^{2}$ MPPC [5, 6], whereas we adopted a $1 \times 1 \mathrm{~mm}^{2}$ MPPC. The pixels aligned in 50- $\mu \mathrm{m}$ pitch which compose the MPPC with $3 \times 3 \mathrm{~mm}^{2}$ sensitive area are bigger than those in $25-\mu \mathrm{m}$ pitch making $1 \times 1 \mathrm{~mm}^{2}$ area. The signal response for the $25 \times 25 \mu \mathrm{m}^{2}$ pixel may be faster than that of the $50 \times 50 \mu \mathrm{m}^{2}$ pixel because of the smaller pixel size for avalanche formation which means that the total capacity of the $25-\mu \mathrm{m}$ pitch is smaller than that of the $50-\mu \mathrm{m}$ pitch. This may contribute to a good timing resolution of $96 \mathrm{ps}$ at FWHM in our experiments.

\section{Materials}

\subsection{Lutetium Fine Silicate}

We used the newly developed inorganic LFS scintillator manufactured by Zecotek Photonics as shown in Figure 1. It has a size of $3 \times 3 \mathrm{~mm}^{2}$ and a high stopping power (TABLEI). Furthermore, it has a short decay time that could result in a good timing resolution. This material has the potential to meet the requirements of next-generation PET systems with fine TOF capability through short decay time $(36 \mathrm{~ns})$. We performed domestic measurements of the light yield of a few scintillators and compared them to the light yield of bismuth germanate (BGO). Because BGO crystal was popular many years ago we compared its light yield to that of LFS and LYSO which are used in present-day PET systems instead of BGO crystal. LFS was found to have a light yield three times greater than that of BGO (Figure 2).

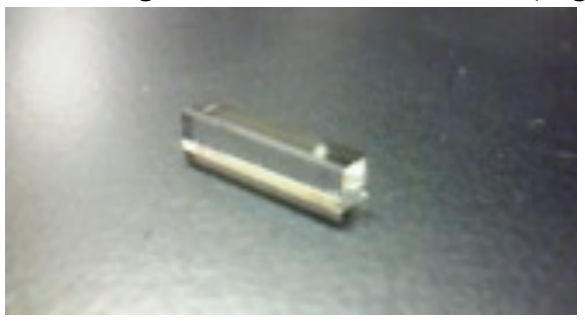

Figure 1 Lutetium Fine Silicate (LFS) is a newly developed inorganic scintillator with a size of $3 \times 3 \times 15$ $\mathrm{mm}^{3}$ 




Figure 2 Light yield of a few inorganic scintillators activated by a ${ }^{22} \mathrm{Na}$ gamma-ray source; the light was collected by a multi-pixel photon counter (MPPC), which had a $25-\mu \mathrm{m}$ pitch.

\begin{tabular}{|c|c|c|c|c|c|}
\hline Material & $\begin{array}{c}\text { Attenuation } \\
\text { length } \\
(\mathrm{cm})\end{array}$ & $\begin{array}{c}\text { Decay } \\
\text { time } \\
\text { constant } \\
\text { (ns) }\end{array}$ & $\begin{array}{c}\text { Maximum emission } \\
\text { of wave length } \\
\text { (nm) }\end{array}$ & \\
\hline LFS & 7.4 & 1.12 & 36 & 416 & 3 \\
\hline LYSO & 7.4 & 1.16 & 44 & 428 & 3 \\
\hline BGO & 7.1 & 1.11 & 300 & 480 & 1 \\
\hline
\end{tabular}

TABLE I.

Properties of inorganic scintillators were taken from the Zecotek Photonics catalogue [3] except for light yield, which was measured in this study.

\subsection{Multi-Pixel Photon Counter (MPPC)}

ADC channels $[\mathrm{ch} / 0.25 \mathrm{pC}]$

The MPPC (S10362-11-025P) manufactured by Hamamatsu K.K. in Japan is a pixelated avalanche photodiode with 1600 pixels in a $1 \times 1 \mathrm{~mm}^{2}$ sensitive area. The size of each pixel is $25 \times 25 \mu \mathrm{m}^{2}$. Since each pixel outputs a signal independent of the number of photons arriving at it, the number of photoelectrons can be counted as the number of fired pixels, as long as the light intensity is sufficiently low to neglect saturation. The gain is $\geq$ to $10^{5}$, which is sufficient for it to be considered as an alternative to the classical photomultiplier tube. MPPC response is not affected by magnetic fields. Therefore, it is expected that PET systems that utilize siliconbased photosensors such as the described MPPC could be combined with magnetic resonance imaging (MRI) systems. This will contribute to new developments in medical imaging. Furthermore, the 


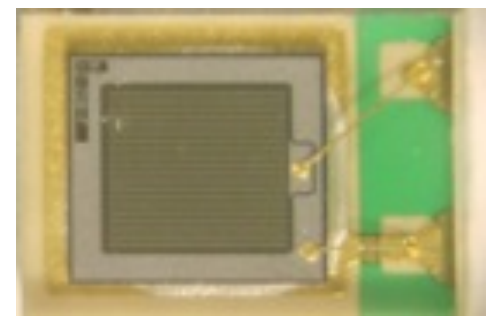

Figure 3 MPPC(S10362-11-025P). A $1 \times 1$ $\mathrm{mm}^{2}$ sensitive area is packed in a surface mount detector (SMD) package of $1.9 \times 2.4 \times$ $0.85 \mathrm{~mm}^{3}$.

LFS crystal emits blue light (416 nm), and the MPPC is sensitive to the blue light region ( 420 $\mathrm{nm}$ ) with good correspondence [4]. MPPC's sensitive area is mounted in a surface mount detector (SMD) package of $1.9 \times 2.4 \times 0.85 \mathrm{~mm}^{3}$ size (Figure 3). The size is suitable for the $3 \times$ $3 \mathrm{~mm}^{2}$ cross section of the LFS crystal used by us. Because of these features, it is expected to be an ideal combination.

\subsubsection{Gain and noise rate}

Figure 4 shows the gain of the 1600 pixel MPPC used in our PET experiment as a linear function of the bias voltage. The breakdown voltage, which is defined as the threshold where the avalanche breakdown occurs, is $73.6 \mathrm{~V}$ and is extracted from the intersection of the line with the horizontal axis.

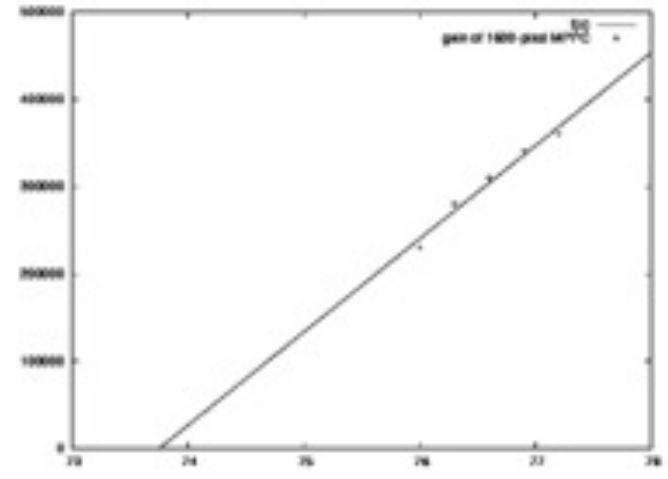

Figure 4 MPPC gain, which has 1600 pixels in a $1 \times 1 \mathrm{~mm}^{2}$ sensitive area, indicates a breakdown voltage of $73.6 \mathrm{~V}$.

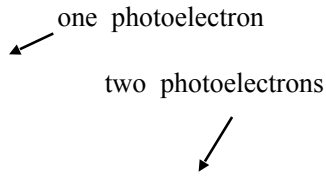

Figure 5 Noise rate was measured by changing the threshold at a bias voltage of 76.1 V. Arrows show the photoelectrons at points of decreasing rate. The signal from the MPPC was amplified by 594.6.

Moreover, the noise rate is another important characteristic parameter of an MPPC. Commonly, semiconducting photon sensors suffer from many noise signals by thermal electrons at room temperature. In case of MPPC, one thermal electron generates a signal equivalent to one photoelectron. To reduce this noise signal donated by a single photoelectron we set the threshold of the discriminator at several photoelectrons or more. Figure 5 shows the dependence of the noise rate on the applied threshold. In particular, the threshold of the realistic PET system is set to be approximately $0.400 \mathrm{MeV}$ or more, depending on the resolution, and the noise signals are negligible.

\section{Experimental Setup}

Both LFS and LYSO crystal's sizes are the same; i.e., $3 \times 3 \times 15 \mathrm{~mm}^{3}$. This size matches the MPPC's package with surface dimensions of approximately $1.9 \times 2.4 \mathrm{~mm}^{2}$. The crystals were

$$
-4-
$$


enclosed in a reflection film (98\% reflectance, Kimoto [7]) and wrapped with black tape to prevent the passage of light. The MPPC has a $1 \times 1 \mathrm{~mm}^{2}$ sensitive area and avalanche photodiodes of 1600 pixels.

LFS (LYSO) and MPPC were directly coupled and connected to a readout circuit. We used two detectors consisting of the MPPC and LFS to take coincidence of annihilation gamma rays. They were aligned and fixed face-to-face with each other. The electronic logic circuit for this measurement is shown in Figure 6.

Readout signals were amplified with an amplifier shaper discriminator (16-ns shaping time) (ASD) [8] and read by a leading-edge discriminator (LED) (Technoland Corporation N-TM415) [9]. ASD outputs the signal, which is amplified and, formed following the low-voltage differential signaling (LVDS) that is above the ASD internal threshold. Therefore, the noise signals are minimized. The gain of the pre-amplifier is $0.8 \mathrm{~V} / \mathrm{pC}$, and the output is fed into the main-amplifier with a gain of 7. ASD is applied to a thin-gap chamber in the ATLAS experiment at LHC/CERN as an amplifier that processes 16 channels in a board. ASD is a relatively fast amplifier; therefore, it will contribute to good timing resolution because of the fast shaping time. Hence, the raw signal from MPPC is shaped as a fast-falling signal. We independently used two channels for signals from the two MPPCs to amplify the signal.

The gains of MPPC are turned so that saturation does not occur in the amplification of ASDs. The ASD is a high-speed amplifier in order to match the 25-ns LHC beam collisions; therefore, the recovery time is shorter than that of the scintillation light.

The threshold values for the LFS-MPPC detectors were set at $200 \mathrm{mV}$, which corresponds to $377 \mathrm{keV}$ in the energy spectrum of the experiment because the PET system detects the $511 \mathrm{keV}$ annihilation gamma rays. According to our energy measurement for $511 \mathrm{keV}$ gamma rays emitted by ${ }^{22} \mathrm{Na}$, their peak fitted by a Gaussian function lies over the $377 \mathrm{keV}$ threshold. Moreover, for the photoelectric effect, the number of photons penetrating into the MPPC surface is constant, whereas for Compton events, the signal should be smaller than that of the events of the photoelectric effect. It is discussed in section 4.2.2.

Both signals from the LED were also used as inputs to the coincidence module. LED signals were fed into a coincidence module (HOSHIN N-024) [10]. The width of MPPC1's LED signal was extended by a gate generator (KAIZU KN1500)[11] before being sent to the coincidence module, such that MPPC2's LED signal fell within the duration. The output from the gate generator was also used as a start signal for timing measurements.

A visual scaler (KAIZU KN1860) [11] was used to count the number of coincident signals for spatial-resolution measurements.

The coincidence module outputs a signal during the rising time of the signal of MPPC2 (Figure 7). Therefore, the coincident signal could be a stop signal in a time-to-digital converter (TDC) (REPIC RPC-060) [12] module. Each signal was fed into TDC, which measures the timing difference by recognizing the timing resolution. The timing resolution was measured with the TDC bin width of 25 ps. Furthermore, a split-start signal, which was delayed by $1 \mu \mathrm{s}$ from the gate generator, was led to TDC, because the TDC module requires clear signals to erase the already stored digital information. When the signal of MPPC 2 arrives before the signal of MPPC 1, TDC stops accepting data. Clear signals avoid this step (Figure 6) because it is considered that a portion of the back-to-back gammas from the ${ }^{22} \mathrm{Na}$ source reach MPPC1 before MPPC2.

The two MPPCs were operated at $75.6 \mathrm{~V}$ so as to have the gain of $2.3 \times 10^{5}$ throughout the experiments. The operating bias of both MPPCs was set from $1 \mathrm{~V}$ to $2 \mathrm{~V}$ over the breakdown voltage.

We used ${ }^{22} \mathrm{Na}$ beta plus decay nuclides as an annihilation gamma-ray source. The beta plus decay is followed by a gamma-ray emission of $1.275 \mathrm{MeV}$, but the gamma ray can be rejected by the coincidence in the face-to-face detector configuration. 
In addition, energy distribution was measured. The signal of MPPC1 is split into two lines before the LED, and one is led to the ADC module for the measured signals. The other, which is fed into the gate generator, is combined with the signal from MPPC2 and passed through LED; then, the coincident signals are used for gating signals in an ADC. The gating signal is set at the time width of $300 \mathrm{~ns}$.

The measurements were performed at room temperature under dark conditions.



\section{Results}

\subsection{Energy resolution}

A charge-integrating analog-to-digital converter (ADC) (REPIC RPC-022) was employed to measure the energy resolution. Signals from both LEDs were fed to the coincidence module, and the output coincident signal generated the gate signal for ADC (Figure 6). The gate width was set to $300 \mathrm{~ns}$ to cover the signal from MPPC. Figure 8 shows the signal from an oscilloscope. Energy distribution is shown in Figure 9. The red line shows the Gaussian fit to the data with an energy resolution of $12.9 \pm 0.2 \%$ in FWHM obtained at $0.511 \mathrm{MeV}$ gamma.

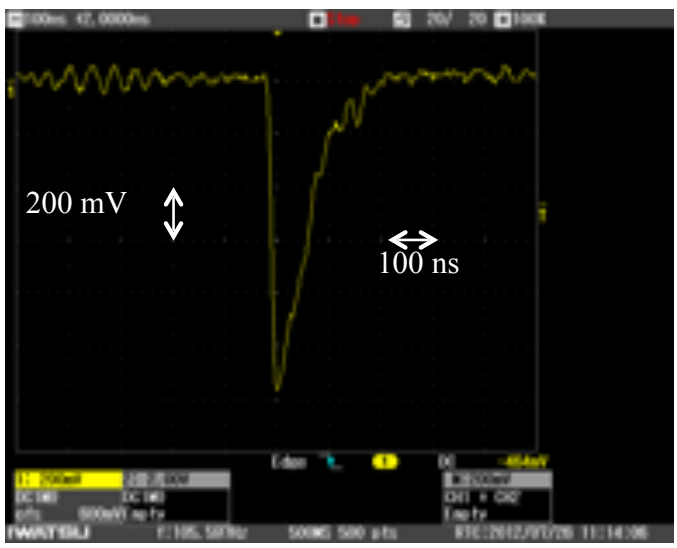

Figure 8 Signal shape from MPPC coupled with LFS.

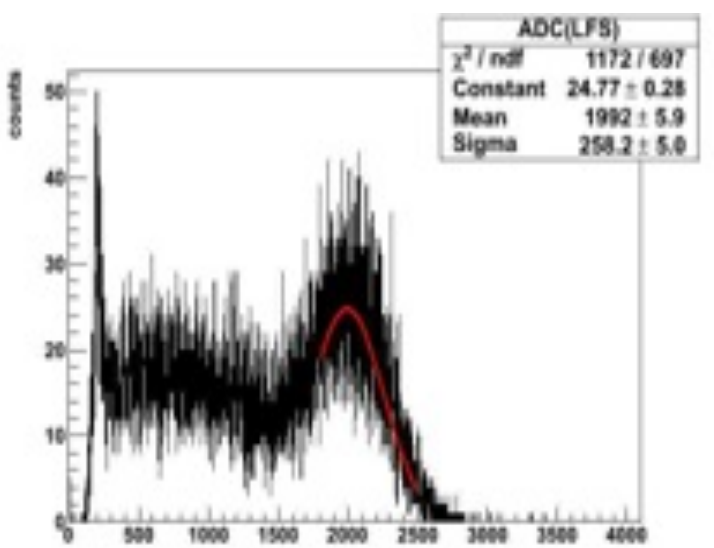

Figure 9 ADC distribution. The $0.511 \mathrm{MeV}$ peak was obtained by gating the back-to-back gamma -6 signals. The energy resolution of $12.9 \%$ was obtained. 


\subsection{Timing resolution}

\subsubsection{Comparison of the timing resolution between LFS and LYSO crystals}

High light yield contributes to a fast timing resolution. Furthermore, MPPC has good timing properties because its own timing resolution is $\sim 100 \mathrm{ps}$ [13]. Many photons reach the sensitive area of MPPC; we could statistically obtain a timing resolution better than 100 ps when MPPC is combined with an LFS inorganic scintillator.

TDC distribution is shown in Figure 10. The vertical and horizontal axes represent the number of counts and TDC channels, respectively. The data were fitted by a Gaussian function, and the timing resolution was evaluated. The sigma of the fitting was $1.64 \pm 0.02$ channels, and it corresponds to $96.2 \pm 1.1 \mathrm{ps}$ at FWHM. In contrast, the LYSO and MPPC coupling resulted in a timing resolution of $111.2 \pm 0.9 \mathrm{ps}$.

The good timing resolution of LYSO with MPPC, whose pixel size is $50 \times 50 \mu \mathrm{m}^{2}$, is reported as $171.5 \pm 0.8 \mathrm{ps}$ [14]. The timing resolution of LFS with MPPC exceeds this result.

A leading-edge discriminator outputs a signal when the rising part of the input signal surpasses the threshold; time jitter affects only the rising time. Therefore, the whole variance of a signal does not influence such timing properties as a part of the tail of a signal. The jitter in the time difference between the signals from two detectors becomes the timing resolution in the TDC module. In addition, our TDC module is operated by common start and stop signals. The stop signal derives a time stamp and draws TDC distribution. Timing resolution is determined by a stop signal, which is generated by the signals taking coincidence. The stop signal is the output when the rising part of a signal exceeds the threshold value. Because of the short jitter in the rising time of a signal, we expect to achieve a good timing resolution with LFS and MPPC.

To obtain evidence of our result for timing resolution, we measured the timing resolution by varying the distance between the ${ }^{22} \mathrm{Na}$ source and $\mathrm{MPPC} 2$, which generates a stop signal, as shown in Figure 11. This means that the stop signal arrives later than the signal for positioning the detector. The detector was moved by $3 \mathrm{~cm}$, which corresponded to four channels on the TDC distribution. Figure 12 shows the mean value of the TDC distribution depending on the distance between the source and MPPC2. The timing resolutions remained constant during these measurements. In this measurement, when the signal of MPPC 2 arrives before that of MPPC1, TDC stops and data are registered until that time is cleared and the new event is encouraged.
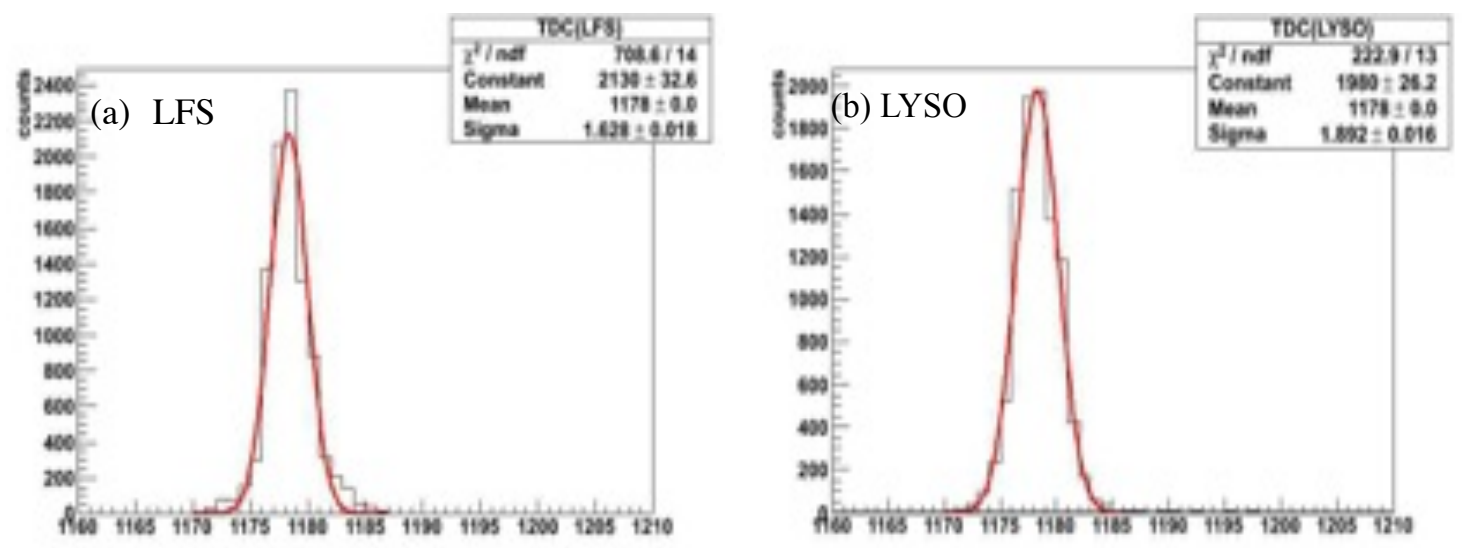

Figure 10 Timing distributions of (a) LFS and (b) LYSO. Each data set was fitted with a Gaussian function and the timing resolution (FWHM) was evaluated. LFS exhibits a better timing resolution than the LYSO crystal.




Figure 11 Shifting the detector moves the peak of the Gaussian fitting to high channels. The 3-cm gap shows how the four channels affect TDC distribution.

Figure 12 shows the Gaussian peak shift that follows linear functions. We recognized that a good timing resolution obtained by the coupling of LFS and MPPC was authentic based on these results.



Figure 12 Mean time is measured as a function of the distance of a pair detector. Changes of the channel complies with the linear function $y$ $=(1.37 \pm 0.17) \mathrm{x}+(1177.10 \pm 0.90)$. The multiplication gradient of 1.37 TDC channels/ $\mathrm{cm}$ times the $3-\mathrm{cm}$ gap gives $4.1 \pm 0.5 \mathrm{ch}$.

\subsubsection{Dependence of thresholds for timing resolution}

We also performed an experiment by changing the threshold value to realize whether the timing resolution will vary under different threshold values. The timing resolution for a threshold of $377 \mathrm{keV}$ was already indicated, which is set to receive signals of the gamma rays undergoing photoelectric phenomena. According to our simulation, the detectors only encounter $30 \%$ of the total annihilation gamma rays in one test. To measure the timing resolution for the energy region above the interaction dominated by the Compton effect, the threshold was changed from $50 \mathrm{mV}$ to $280 \mathrm{mV}$ for each detector simultaneously. These values are equivalent to $94 \mathrm{keV}$ and $528 \mathrm{keV}$, respectively. The timing resolution is depicted in Figure 13. Both LFS and LYSO show the same dependency on threshold values, although the absolute values are different. Their curves flatten for threshold values greater than $377 \mathrm{keV}$. In addition, the timing resolution becomes better in lower threshold values.

It is commonly known that high light yield contributes to a good timing resolution. However, our timing resolution results including gamma rays undergoing Compton scattering are better than those with gamma rays detected solely as a result of the photoelectric effect. In this paper, the timing property was settled by fluctuation of the TDC stop signal, which is derived from the output signals of the coincidence module. The coincidence module outputs a signal pulse relative to the timing of the down edge of the signal pulse from MPPC 2 when the coincidence timing is determined, as shown in Figure 7. Therefore, the timing resolution is fixed by the signal rising time. The timing is affected by the stochastic nature of the photon production in the crystal and the arrival at the MPPC. However, this thesis considers only one photoelectron. An MPPC detects photons of approximately 100 p.e./MeV in our experiment; therefore, this logic is notvalid. In addition, we shifted the two detectors in opposite directions to change the timing an ${ }^{5}$ achieved the same timing resolution, which indicates the stability of our results. 


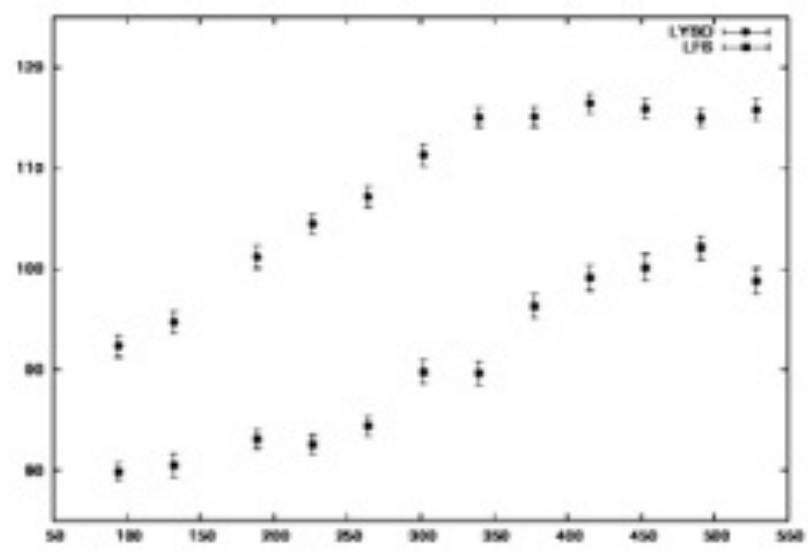

Figure 13 Timing resolution as a function of the threshold. The low thresholds indicate a better timing resolution than that of Figure 10. However, considering to the PET system, we should set the threshold at $0.511 \mathrm{MeV}$, because the PET system deals with only $0.511-\mathrm{MeV}$ annihilation gamma rays.

\subsubsection{Mechanisms of jitter and timing resolution}

The low threshold results in a good timing resolution according to our measurement. The mechanism is explained by the nature of the signal output from MPPC. The fast rising time contributes to a good timing resolution. The jitter of the rising part of signals constructs TDC distribution, and short jitter affects small sigma while fitting them by a Gaussian function. In case of low thresholds, the jitter of the lower part of a signal is measured. On the other hand, in case of high thresholds, the jitter of the higher part of the signal is determined. Based on this condition, the jitter of the lower part of a signal may be shorter than that of the higher part. Figure 14 (a) shows signals that draw three different heights, assuming that many signals arrive at the detector in the experiment. At low threshold, the signals comprise short jitters, whereas at high threshold, they comprise long jitters. Therefore, the rising part of a signal should be considered positively.

(a)

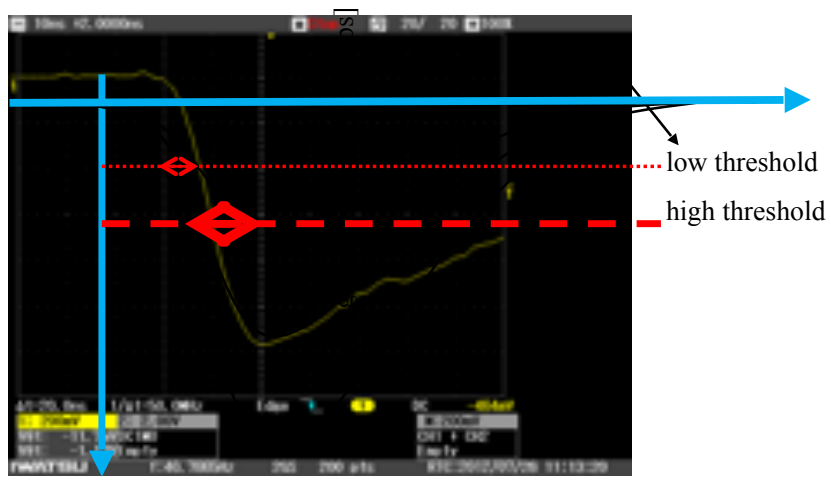

(b)

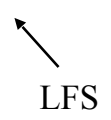

threshold [keV]

Figure 14 (a) Different jitters are constructed by low and high thresholds. Low threshold could construct a short jitter which achieves a timing resolution better than that of high threshold. (b) The rising part of a signal is shown as an expansion of figure 8.

time 


\subsection{Spatial resolution}

Spatial resolution is important in evaluating PET system performance. We counted the number of coincidences from the two LFSs when the position of the source location, set on the stage driven by a micrometer, was changed by $0.50 \mathrm{~mm}$ (from $-5 \mathrm{~mm}$ to $+5 \mathrm{~mm}$ ) along the line perpendicular to the one that connects the two LFSs, as shown in Figure 15. The distance between LFS and the source was set to $9 \mathrm{~cm}$. To construct a new PET system, we need a resolution on the order of millimeters; thus, it is important to know the achievable resolutions of the system.

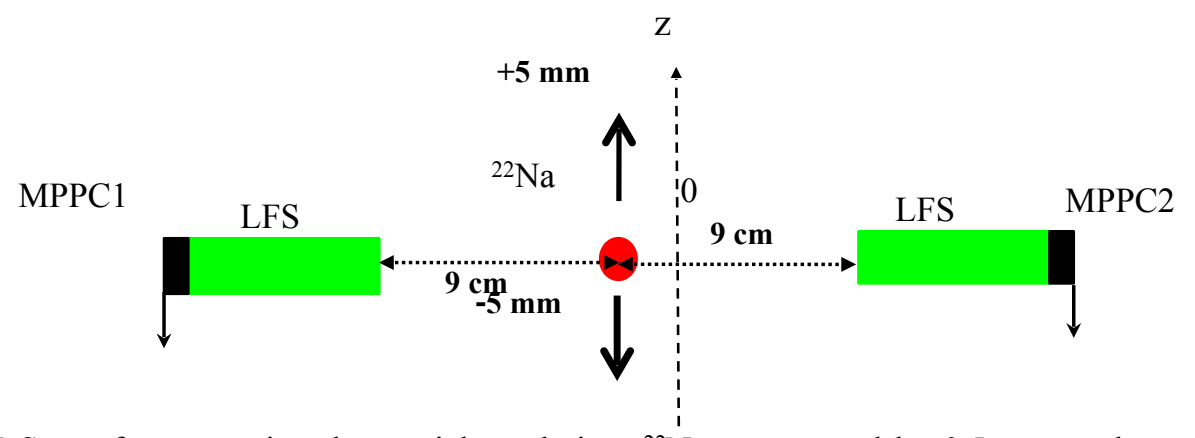

Figure 15 Setup for measuring the spatial resolution; ${ }^{22} \mathrm{Na}$ was moved by $0.5 \mathrm{~mm}$ at the center of the detectors along the orthogonal $\mathrm{z}$ line. Coincident signals were counted at each point.

Figure 16 (a) shows the number of coincident events as a function of the source position by the dots, where the solid line is the fitting result of the Gaussian curve. The spatial resolution was $1.90 \pm 0.09 \mathrm{~mm}$ which is consistent with the $3 \times 3 \mathrm{~mm}^{2}$ size of the scintillator cross section. To recognize this result, we performed a GEANT4 simulation assuming the same experimental conditions as with our own code. First, the geometry of the detector was constructed with two LSOs placed face-to-face because we cannot know the particular components of an LFS crystal. In addition, the spatial resolution was measured to move a ${ }^{22} \mathrm{Na}$ source at the center of the detectors. The situation is also described in Figure 14. According to our simulation [Figure 16 (b)], the spatial resolution depends on the scintillator size; therefore, the results are consistent with expectations.

(a) measured spatial resolution

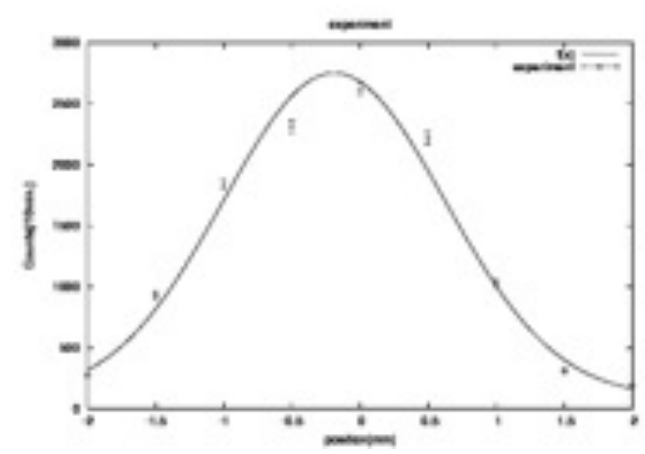

(b) simulated spatial resolution

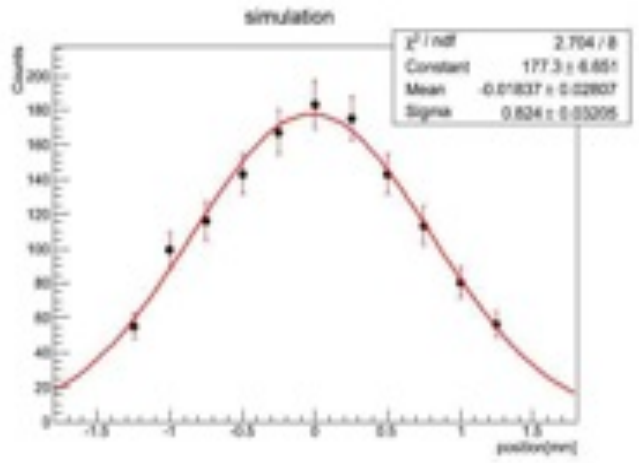

Figure 16 (a) Spatial resolution was measured at a 9-cm distance between the two LFSs. The ${ }^{22} \mathrm{Na}$ source position is changed by $0.50 \mathrm{~mm}$ to the direction perpendicular to the gamma line. Numbers of coincidence events are plotted as a function of the movement of the source. The result of the Gaussian fitting was $1.90 \pm 0.09 \mathrm{~mm}$ in FWHM. (b) Simulation results of the Gaussian fit, which show $1.93 \pm 0.07$ $\mathrm{mm}$ in FWHM for spatial resolution. 


\section{Discussion}

In this study, timing resolution is remarkably affected by small fluctuations in the rising time of the signal from detectors. A good timing resolution is expected from the large number of photons generated inside a scintillator crystal when it is statistically dominant. In addition, electronic components that have a fast response will contribute to a good timing resolution, such as photosensors, amplifiers and computer-aided measurement and control (CAMAC) modules that are used for data acquisition systems in the field of high-energy physics.

We employed a leading-edge discriminator (LED). This module outputs the signal when the rising part of the input signal exceeds the threshold value. The timing property is affected only by the rising part of the signal. Some teams measuring the timing resolution of PET systems employ a constant fraction discriminator (CFD); however, this study does not mention the falling part of the signals. As good timing resolution is achieved by fast rising time of signals with an LED, we show a good solution for the next-generation PET system. LFS indeed has fast-decay time. Commonly, with a scintillator that produces fast decay time, fast rising time can be assumed. Therefore, a CFD will not be required if we deal with the rising part of the signals from LFS.

\section{Conclusions}

We measured the timing resolution with an LFS inorganic scintillator and an MPPC photosensor. Compared with the LYSO crystal in the MPPC coupling, better timing resolution was obtained with an LFS smaller than 100 ps at FWHM, which corresponds to a spatial resolution of $3 \mathrm{~cm}$; hence, LFS could be a potential substitute for LYSO. Because LFS has a fast rising time, time jitter could be minimized. A good timing resolution results in clearer images by preventing noise. In addition, LFS crystal is currently less expensive than any other inorganic scintillator crystal. These facts indicate that high-performance, next-generation PET systems could be constructed at low cost. Higher performance and less expensive clinical equipment could contribute to better cancer diagnosis.

\section{References}

1. J. S. Karp, S. Surti, M. E. Daube-Witherspoon, G. Muehllehner, Benefit of Time-of-Flight in PET: Experimental and Clinical Results, J. Nucl. Med., 49 (2008), 462

2. W.W.Moses, Time-of-flight in PET revisited, IEEE Trans. Nucl. Sci., 50 (2003), 1325

3. Zecotek Photonics Inc., http://www.zecotek.com/

4. Hamamatsu Photonics K. K., http://jp.hamamatsu.com/

5. G. Martin, G. Erika, K. Valentin, S. C. Hans-Christian, T. Alexander, T. Adel, Application of multipixel photon counter to positron emission tomography, Proceedings of the Nuclear Science Symposium Conference Record (NSS), p. 3119, Dresden, Germany, Oct. 19-25, 2008

6. C. L. Kim, G. C. Wang Dolinsky, IEEE Trans. Nucl. Sci., 56 (2009), 2580

7. Kimoto Co. Ltd., http://www.kimoto.co.jp/

8. O. Sasaki and M. Yoshida, ASD IC for the Thin Gap Chambers in the LHC Atlas Experiment, IEEE Trans. Nucl. Sci., 46 (1999), p.1871

9. Technoland Co. Ltd., http://www.tcnland.co.jp/

10.Hoshin Electronics Co., Ltd., http://www.kagaku.com/hoshin/

11. Kaizu Works Co., http://www.kaizuworks.co.jp/

$$
-11-
$$


12. REPIC Co., Ltd., http://www.repic.co.jp/

13. R. Pestotnik, et al., Silicon photo-multipliers as photon detectors for PET, Nucl. Instrum. Meth. A623 (2010), 594-596

14. S. Seifert, R. Vinke, H. T. van Dam, H. Lohner, P. Dendooven, F. J. Beekman, D. R. Schaart, Ultra precise timing with SiPM-based TOF PET scintillation detectors, Proceedings of the Nuclear Science Symposium Conference Record (NSS/MIC), p. 2329, Orlando, FL, Oct. 24 2009-Nov. 1, 2009 